\title{
Unknown Dosage Form Category
}

National Cancer Institute

\section{Source}

National Cancer Institute. Unknown Dosage Form Category. NCI Thesaurus. Code C150001.

The type of pharmaceutical dose form is unknown, or has unspecified or variable physical characteristics. 